in vivo $35: 1185-1189(2021)$

doi:10.21873/invivo.12367

\title{
Progression After Molecular Targeted Agents: Hepatic Arterial Changes and Transarterial Chemoembolization in Hepatocellular Carcinoma
}

\author{
NORITAKA MATSUDA ${ }^{1}$, NORIHIRO IMAI ${ }^{1}$, TEIJI KUZUYA ${ }^{2}$, KENTA YAMAMOTO $^{1}$, TAKANORI ITO $^{1}$, \\ YOJI ISHIZU ${ }^{1}$, TAKASHI HONDA ${ }^{1}$, MASATOSHI ISHIGAMI ${ }^{1}$ and MITSUHIRO FUJISHIRO ${ }^{1}$ \\ ${ }^{1}$ Department of Gastroenterology and Hepatology, Nagoya University Graduate School of Medicine, Aichi, Japan; \\ ${ }^{2}$ Department of Gastroenterology and Gastroenterological Oncology, Fujita Health University, Aichi, Japan
}

\begin{abstract}
Background/Aim: The purpose of this study was to determine changes in the hepatic arteries after treatment with a molecular targeted agent (MTA), and evaluate the safety and efficacy of transarterial chemoembolization (TACE) as a post-MTA treatment in patients with hepatocellular carcinoma (HCC). Patients and Methods: The cases of 33 patients with intermediate HCC treated with MTA and TACE were studied retrospectively. The hepatic arteries, and the safety and efficacy of TACE were evaluated before and after MTA treatment. Results: Following longterm MTA treatment, the diameters of hepatic arteries decreased significantly, while there was no difference in the diameters of the splenic artery or the portal vein. No significant adverse events were observed due to TACE after MTA; however, the therapeutic effect of TACE was limited after MTA treatment. Conclusion: This study demonstrated that the diameters of hepatic arteries were significantly smaller than those before MTA induction, suggesting ischemic effects and tumor vessel "normalization" by MTA treatment. Although TACE can be performed as a post-MTA treatment without lowering the hepatic reserve or causing serious complications, its therapeutic effect is limited.
\end{abstract}

The clinical importance of molecular targeted agents (MTA) for the treatment of hepatocellular carcinoma (HCC) is rapidly increasing, and it is widely recommended as a

This article is freely accessible online.

Correspondence to: Norihiro Imai, MD, Ph.D., Department of Gastroenterology and Hepatology, Nagoya University Graduate School of Medicine, 65 Tsurumai-cho, Showa-ku, Nagoya-shi, Aichi-ken 466-8560, Japan. Tel: +81 527442169, Fax: +81 527442178, e-mail: norihiro.imai@gmail.com

Key Words: Sorafenib, lenvatinib, hepatic artery, transarterial chemoembolization, hepatocellular carcinoma. standard treatment not only for HCCs with vascular invasion or distant metastasis, but also for HCCs that are refractory to transarterial chemoembolization (TACE) (1). Among MTAs currently available for HCC, sorafenib targets vascular endothelial growth factor (VEGF) and plateletderived growth factor receptor (PDGFR), while lenvatinib targets vascular endothelial growth factor receptor (VEGFR). Therefore, both are known to exhibit antitumor effects by suppressing angiogenesis in the tumor (2).

By suppressing tumoral angiogenesis, MTA treatment is expected to "normalize" blood vessels by modifying interstitial pressure and vascular permeability $(3,4)$. Therefore, enhanced therapeutic effects of TACE post-MTA treatment are expected. However, there are no reports on changes in blood flow to the liver after long-term MTA treatment or the therapeutic effect of TACE on HCC after MTA treatment.

The purpose of this study was to determine (i) changes in the hepatic arteries after long-term MTA treatment, and (ii) the safety and efficacy of TACE as a post-MTA treatment.

\section{Patients and Methods}

From August 2011 to August 2020, 261 patients with HCC were treated with MTAs at the Nagoya University Hospital, Aichi, Japan. Among them, 59 patients were treated with MTA for TACE-refractory HCC, and TACE was reintroduced in 33 patients due to tumor progression after MTA treatment. Among the latter group of patients, 26 patients were treated with sorafenib, and 7 patients were treated with lenvatinib. The average MTA treatment period prior to the reintroduction of TACE was 516 (14-2411) days. The average age of these patients was 68.8 (44-89) years. In the same group, all patients were classified as having Barcelona Clinic Liver Cancer (BCLC) stage B. We compared the diameters of hepatic arteries before and after MTA treatment. Since patients were reintroduced TACE due to tumor progression, the diameters of hepatic arteries were also evaluated at the time when best MTA tumor response was achieved. The safety and efficacy of TACE were evaluated before and after MTA administration.

Contrast-enhanced computed tomography was performed prior to each TACE procedure. To analyze the vascular changes, the 
maximum cross-sectional diameters of the blood vessels were measured using images acquired during the arterial and portal vein phases. Measurements were made individually by two gastroenterologists. TACE after MTA treatment was performed according to standard procedures $(5,6)$, typically after 3-7 days of MTA withdrawal. The safety of TACE was evaluated using the Common Terminology Criteria for Adverse Events v5.0 (CTCAE v5.0). Tumor markers and Child-Pugh Score (CPS) were evaluated before, 1 week after, and 1 month after each TACE procedure. The level of statistical significance was set at $p<0.05$. All statistical analyses were performed using GraphPad Prism (version 8.4.3). This study was a retrospective study and was approved by the Ethics Committee of Nagoya University Hospital (2019-0031).

\section{Results}

Following long-term MTA treatment (average=516 days), the diameters of hepatic arteries, from the common hepatic artery to the peripheral arteries, were significantly smaller than those before MTA treatment (Figure 1A-E). On the other hand, no difference was observed in the diameters of the splenic artery or portal vein (Figure $1 \mathrm{~F}$ and $\mathrm{G}$ ). Importantly, same changes were also observed at the time when best MTA tumor response was achieved (average 57 days) (Figure $1 \mathrm{C}-\mathrm{G}$ ). These results imply that after MTA treatment, the diameters of hepatic arteries relatively decreased due to tumor ischemia and "normalization" of tumor blood vessels in the liver.

Regarding the efficacy of TACE after MTA, there were no decreases in alpha fetoprotein (AFP) levels or in des-gammacarboxy prothrombin (DCP) after the treatment (Figure 2A and B). CPS before TACE was mildly elevated (0.7), even with tumor progression after long-term MTA treatment (Figure 2C). The increase in CPS after TACE was greater when it was performed after MTA compared to that before MTA (0.5 vs. $0.9)$, but the CPS returned to baseline one month after TACE (Figure 2C). The adverse effects of TACE before MTA were cholangitis (Grade 3) in 1 case, cholecystitis (Grade 3) in 2 cases, fever (Grade 3) in 1 case, and localized peritonitis (Grade 3) in 1 case. Meanwhile, the adverse effects of TACE after MTA were ascites (Grade 3) in 1 case, and cholangitis (Grade 3 ) in 2 cases. No other serious complications were observed for both, TACE before MTA and TACE after MTA.

\section{Discussion}

Following long-term MTA treatment, the diameters of hepatic arteries were smaller than those before MTA induction, while there was no difference in the diameters of the splenic artery or the portal vein. The increase in CPS was minimal, even with tumor progression after long-term MTA treatment in patients with TACE-refractory HCC. Although the therapeutic effect of TACE after MTA treatment was limited, it can be performed without lowering the hepatic reserve or causing serious complications.
The decrease in the diameters of hepatic arteries has previously been reported as a vascular change due to combined treatment of TACE and sorafenib (7). They concluded that combination therapy with TACE and sorafenib may reduce serum VEGF and inhibit angiogenesis. Notably, they did not observe the same hepatic arterial changes in patients treated with TACE alone. In contrast, we found that the diameters of the common hepatic artery and further peripheral arteries were smaller after MTA administration alone. These results may suggest that MTA treatment induces tumor ischemia and "normalization" of tumor vessels, resulting in a relative decrease in blood flow to the liver. The expected effects of VEGF inhibitor administration are "normalization" of abnormal tumor blood vessels by maintaining a balance of suppression and promotion of angiogenesis in the tumor, modification of intra-tumoral interstitial pressure, and changes in vascular permeability $(3,4)$. Thus, we hypothesized that through these vascular changes in the tumor, the efficiency of TACE may be enhanced. However, this study showed that the therapeutic effect of TACE was limited after MTA treatment. This might be because TACE was performed upon tumor progression after MTA treatment.

Recent advances in MTA treatment have improved the prognosis of patients with advanced hepatocellular carcinoma (8-10). However, the use of MTAs in treatment is limited due its various adverse effects. This suggests the importance of HCC care after MTA treatment. However, no post-MTA treatment guidelines have been established thus far. In this study, the effect of TACE as a post-MTA treatment is limited and its effect on prognostic improvement cannot be stated; however, it can be performed without lowering hepatic reserve or causing serious complications.

This study has some limitations. First, the number of patients was limited. Second, only patients who underwent TACE were enrolled in this study. Third, this study had a retrospective design because the presence of bias related to the selection of patients for HCC treatment cannot be ruled out.

\section{Conclusion}

This study demonstrated that in post-MTA TACE treatment, the diameters of hepatic arteries were significantly smaller than those before MTA induction, due to ischemic effects and tumor vessel "normalization" by MTA treatment in patients with HCC. Although TACE can be performed postMTA treatment without serious complications, its therapeutic effect is limited.

\section{Conflicts of Interest}

T.K.: Honoraria from Bayer, Eisai, Eli Lilly Japan. Research funding from Eisai. 

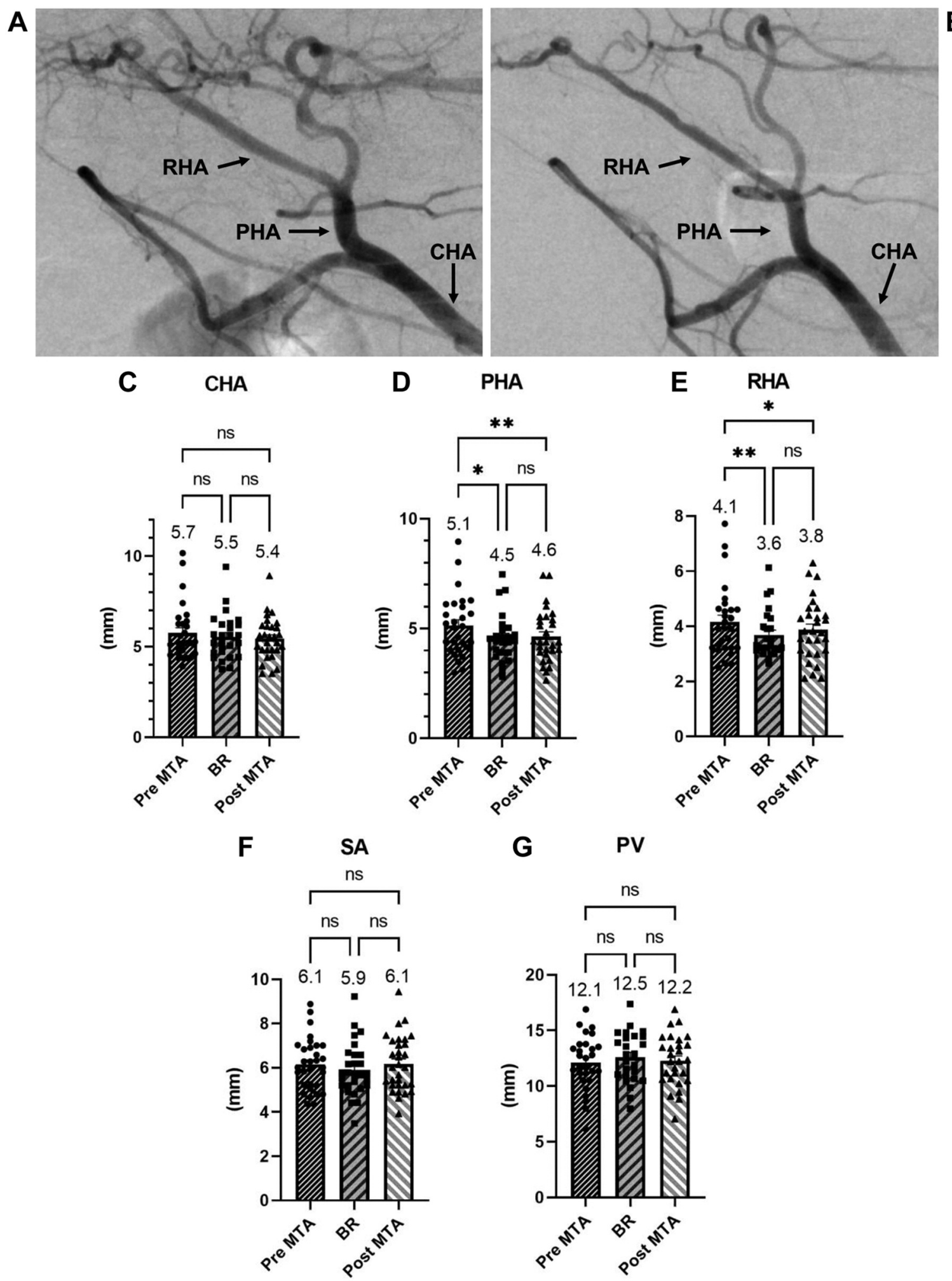

Figure 1. Vascular changes before and after treatment with molecular targeted agents. Representative case of vascular change after treatment with a molecular target agent (MTA). Images show angiographies performed prior to MTA treatment (A) and one year after MTA treatment (B). (C-G) Bars represent the diameters of vessels before and after MTA treatment and at the time when best MTA tumor response was achieved. $p$-Values were calculated using paired t-test with Bonferroni correction. BR, Best response; CHA, common hepatic artery; PHA, proper hepatic artery; RHA, right hepatic artery; SA, splenic artery; PV, portal vein; $n s$, not significant; *p<0.05; **p<0.01. 

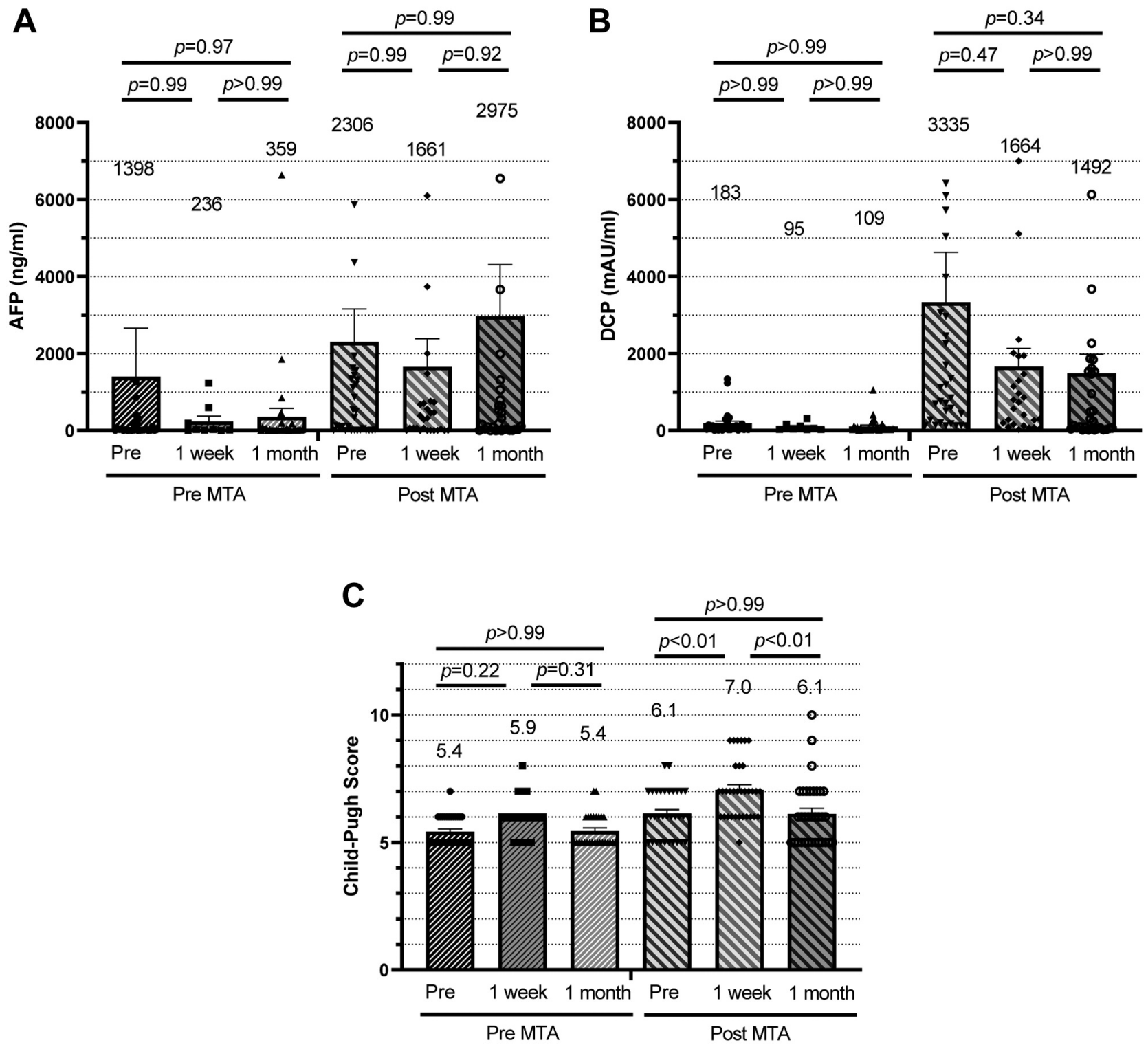

Figure 2. Treatment effect of transarterial chemoembolization before and after treatment with molecular targeted agents (MTA). Bars represent plasma concentrations of AFP (A), DCP (B) and the Child-Pugh Score, one day before (pre), one week after and one month after each TACE treatment. $p$-Values were calculated by Tukey's multiple comparisons. AFP, Alpha fetoprotein; DCP, des-gamma-carboxy prothrombin.

\section{Authors' Contributions}

N.M., N.I., T.K., K.Y., T.I., Y.I., T.H., M.I. and M.F. performed the research. N.M. and N.I. designed the research study. N.M. and N.I. analysed the data. N.I. wrote the paper.

\section{References}

1 Cheng AL, Amarapurkar D, Chao Y, Chen PJ, Geschwind JF, Goh KL, Han KH, Kudo M, Lee HC, Lee RC, Lesmana LA, Lim HY, Paik SW, Poon RT, Tan CK, Tanwandee T, Teng G and Park JW:
Re-evaluating transarterial chemoembolization for the treatment of hepatocellular carcinoma: consensus recommendations and review by an International Expert Panel. Liver Int 34(2): 174-183, 2014. PMID: 24251922. DOI: 10.1111/liv.12314

2 Kudo M, Finn RS, Qin S, Han KH, Ikeda K, Piscaglia F, Baron A, Park JW, Han G, Jassem J, Blanc JF, Vogel A, Komov D, Evans TRJ, Lopez C, Dutcus C, Guo M, Saito K, Kraljevic S, Tamai T, Ren M and Cheng AL: Lenvatinib versus sorafenib in first-line treatment of patients with unresectable hepatocellular carcinoma: a randomised phase 3 non-inferiority trial. Lancet 391(10126): 1163-1173, 2018. PMID: 29433850. DOI: 10.1016/ S0140-6736(18)30207-1 
3 Jain RK: Normalization of tumor vasculature: An emerging concept in antiangiogenic therapy. Science 307(5706): 58-62, 2005. PMID: 15637262. DOI: 10.1126/science.1104819

4 Liu K, Zhang X, Xu W,Cheng J, Yu J, Gamble JR and McCaughan GW: Targeting the vasculature in hepatocellular carcinoma treatment: Starving versus normalizing blood supply. Clin Transl Gastroenterol 8(6): e98, 2017. PMID: 28617447. DOI: $10.1038 / \operatorname{ctg} .2017 .28$

5 Imai N, Ishigami M, Ishizu Y, Kuzuya T, Honda T, Hayashi K, Hirooka $\mathrm{Y}$ and Goto H: Transarterial chemoembolization for hepatocellular carcinoma: a review of techniques. World $\mathrm{J}$ Hepatol 6(12): 844-850, 2014. PMID: 25544871. DOI: 10.4254/ wjh.v6.i12.844

6 Imai N, Katano Y, Kuzuya T, Honda T, Hayashi K, Ishigami M, Itoh A, Hirooka Y and Goto $\mathrm{H}$ : An increase in lesion density can predict lower local recurrence after transarterial chemoembolization in patients with hepatocellular carcinoma. Hepatogastroenterology 60(125): 965-970, 2013. PMID: 23425812. DOI: $10.5754 /$ hge 121229

7 Chen L, Zheng Y, Zhang H, Pan H, Liu Q, Zhou X, Wei W, Liu Y, Zhen M, Wang J, Zhou J and Zhao Y: Comparative analysis of tumor-associated vascular changes following TACE alone or in combination with sorafenib treatment in HCC: a retrospective study. Oncol Lett 16(3): 3690-3698, 2018. PMID: 30127979. DOI: $10.3892 / \mathrm{ol} .2018 .9055$
8 Kuzuya T, Ishigami M, Ito T, Ishizu Y, Honda T, Ishikawa T, Hirooka $\mathrm{Y}$ and Fujishiro $\mathrm{M}$ : Clinical characteristics and outcomes of candidates for second-line therapy, including regorafenib and ramucirumab, for advanced hepatocellular carcinoma after sorafenib treatment. Hepatol Res 49(9): 10541065, 2019. PMID: 31033165. DOI: 10.1111/hepr.13358

9 Kuzuya T, Ishigami M, Ito T, Ishizu Y, Honda T, Ishikawa T and Fujishiro M: Favorable radiological antitumor response at 2 weeks after starting lenvatinib for patients with advanced hepatocellular carcinoma. Hepatol Res 50(3): 374-381, 2020. PMID: 31721363. DOI: 10.1111/hepr.13452

10 Tanaka T, Kuzuya T, Ishigami M, Ito T, Ishizu Y, Honda T, Ishikawa T and Fujishiro M: Efficacy and safety of sorafenib in unresectable hepatocellular carcinoma with bile duct invasion. Oncology 98(9): 621-629, 2020. PMID: 32434180. DOI: $10.1159 / 000507051$

Received December 9, 2020

Revised January 15, 2021

Accepted January 18, 2021 